\title{
Hybrid Denoising Method for Removal of Mixed Noise in Medical Images
}

\author{
J UMAMAHESWARI \\ Research Scholar, Department of Computer Science \\ Dr.G.R.D. College of Arts and Science \\ Coimbatore, Tamil Nadu, India.
}

\author{
Dr.G.RADHAMANI \\ Director, Department of Computer Science \\ Dr.G.R.D. College of Arts and Science \\ Coimbatore, Tamil Nadu, India.
}

\begin{abstract}
Nowadays, Digital image acquisition and processing techniques plays a very important role in current day medical diagnosis. During the acquisition process, there could be distortions in the images, which will negatively affect the diagnosis images. In this paper a new technique based on the hybridization of wavelet filter and center weighted median filters is proposed for denoising multiple noise (Gaussian and Impulse) images. The model is experimented on standard Digital Imaging and Communications in Medicine (DICOM) images and the performances are evaluated in terms of peak signal to noise ratio (PSNR), Mean Absolute Error (MAE), Universal Image Quality Index (UQI) and Evaluation Time (ET). Results prove that utilization of center weighted median filters in combination with wavelet thresholding filters on DICOM images deteriorates the performance. The proposed filter gives suitable results on the basis of PSNR, MSE, UQI and ET. In addition, the proposed filter gives nearly uniform and consistent results on all the test images.
\end{abstract}

Keywords- Gaussian noise; impulse noise; UQI; Wavelet filter; CWM; hybrid approach.

\section{INTRODUCTION}

Many scientific datasets are contaminated with noise, either because of the data acquisition process, or because of naturally occurring phenomena. Pre-processing is the first step in analyzing such datasets. There are several different approaches to denoise images. The main problem faced during diagnosis is the noise introduced due to the consequence of the coherent nature of the image capture. In image processing applications, linear filters tend to blur the edges and do not remove Gaussian and mixed Gaussian impulse noise effectively [7], [8]. Inherently noise removal from image introduces blurring in many cases. These noises corrupt the image and often lead to incorrect diagnosis. Gaussian noise is an additive noise, which degrades image quality that originates from many microscopic diffused reflections leads to discriminate fine detail of the images in diagnostic examinations [1], [2], [3]. Thus, denoising these noises from a noisy image has become the most important step in medical image processing.

The most common type of noise is generated by the detector [6],[8]. Thermal fluctuations is one type of disturbance occurred due to many interconnected electronics components which has a Gaussian Probability Density
Function (PDF), from the contributions of many independent signals, PDF results in a signal with a Gaussian PDF [11],[12]. Many methods have been available for noise reduction [13], [14], [9]. The existing filters used for mixed noise reduction techniques includes median filter, center weighted median filter and wavelet filters. Nowadays, the uses of wavelet based denoising techniques have gained more attention by researchers [10].

In this work a fusion technique is proposed to find the best possible solution, so that after denoising PSNR, MSE, UQI and ET values of the image are optimal. The proposed method is based on wavelet transform and center weighted median filtering, which exploits the potential features of the combination of both wavelet and center weighted median.

This paper is organized as follows. Section 2 discusses the wavelet based thresholding method for denoising Gaussian noise. Section 3 describes the center weighted median filter for denoising impulse noise. The proposed methodology is explained in Section 4. Experimental results are given in Section 5. Finally Conclusion and reference are discussed in Section 6.

\section{WAVELET BASED THRESHOLDING}

The following figure 1 shows the wavelet denoising steps:

- Apply wavelet transform to the noisy image to produce the noisy wavelet coefficients.

- $\quad$ Select best appropriate threshold limit at each level by using threshold method (hard or soft thresholding) to remove the noises. Here soft thresholding is used for removal of noise.

- Inverse wavelet transform [5] is applied to thresholded wavelet coefficients to obtain a denoised image.

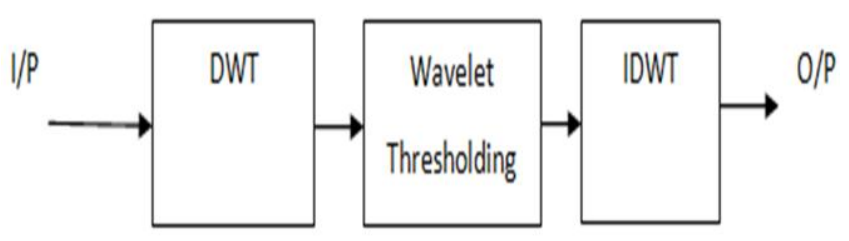

Figure 1. Denoising using wavelets transform filtering 


\section{A. Wavelet Representation of Image}

During transmission the image $f$ is corrupted by white Gaussian noise with independent and identically distributed by mean, and standard deviation. The noisy image received is $g_{i j}$ $=f_{i j}+\mathrm{s} n_{\mathrm{ij}}$. When estimate the image signal $f$ from noisy observations $g_{i j}$, PSNR and UQI is maximum as well as MSE and ET should be minimum. These methods use a threshold and determine the wavelet coefficients. There are two types of thresholding for removal of noise, namely the hard thresholding method and the soft thresholding method [2],[10].

\section{1) Soft Thresholding Method}

Generally Hard thresholding is discontinuous, to overcome this Donoho [8] introduced the soft thresholding method. If the absolute value of a coefficient is less than a threshold, then is assumed to be 0 , otherwise its value is shrunk by threshold.

This removes the discontinuity, but degrades all the other coefficients which tend to blur the image. In the soft thresholding method, there are deviations between image coefficients and threshold coefficients which directly influence the accuracy of the reconstructed image.

\section{Center Weighted Median Filter}

When one give more weight to the central value of the window a special case of weighted median filters called the Center Weighted Median filter will be produced, and thus it is easier to design and implement the general weighted median filters. For the discrete-time continues-valued of $K$ input samples in $M \times N$ window $W$ at point $\left(n_{1}, n_{2}\right), n_{1}=1, \ldots, N_{1}, n_{2}=1$, $\ldots, N_{2}, u\left(n_{1}, n_{2}\right)=[u(1), \ldots, u(K)]$, the output $y$ of center weighted Median filter of $K$ samples is given by [5],

$$
y\left(\boldsymbol{n}_{1,} \boldsymbol{n}_{2}\right)=\operatorname{MED}[u(1), \ldots . ., u(K), 2 l \ldots . . u(1) K \in W]
$$

Where, $l$ is a non-negative integer. When $l=0$, the CWM filter becomes the median filter, and when $2 l+1$ is greater than or equal to the window size, it becomes the identity filter. The statistical properties of center weighted median filters have been studied to evaluate the noise suppression, edge and detail, e.g. fine lines, preservation characteristics, while the study of the deterministic properties includes root sets and convergence behavior of the filters in time domain. For identically and independently distributed inputs $F(n)$, the output distribution function $P_{c w m}(n)$ of the center weighted median with $K$ number of samples, $K=2 k+1$, and center weight $L=2 l+1$ is given[9],

$P_{C W M}=\sum_{k+1-l}^{2 k}\left(\frac{2 k}{i}\right) F(n)^{i+1}(l-F(n))^{2 k-i}+\sum_{k+1+l}^{2 k}\left(\frac{2 k}{i}\right) F(n)^{i}(l-F(n))^{2 k+1-l}$

Obviously, a center weighted median filter with a larger central weight performs better in detail preservation than with a smaller central weight. The central weight should be carefully selected depending on the characteristics of the input image and its noise. The advantage of center weighted median filter is to reduce noise and to preserve fine details.

\section{PROPOSED METHOdODLOGY}

In the hybrid work, two techniques namely, wavelet thresholding and center weighted median filters are combined to form a hybrid denoising model. These techniques are used

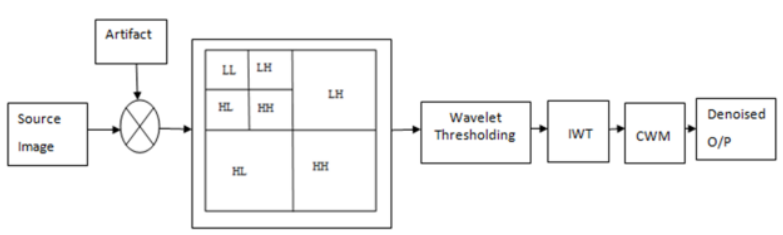

to suppress the mixed noise (Gaussian and impulse noise). The figure 2 shows the proposed method for denoising mixed noise in medical domain.

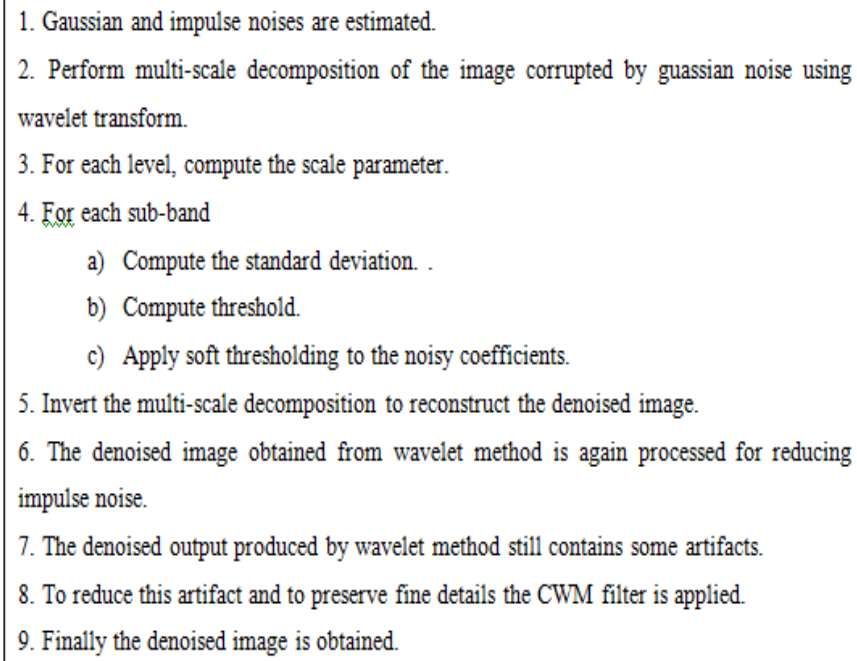

Figure 2. Proposed methods for denoising mixed noise in medical images

Wavelets have made quite a splash in the field of image processing. Proposed model is the newly designed hybridized one as shown in figure 2 . In this model, the image is denoised first with wavelet decomposition into four sub-bands using haar wavelet filters. In the next level the wavelet based soft thresholding is applied on all the sub-bands. The shrink wavelet co-efficient is a soft thresholding is applied. It is used for suppressing the Gaussian noise. Resultant coefficients are used for image reconstruction with IWT. The results obtained after thresholding are then used to reconstruct the image. In the last level, center weighted median filter is used to remove impulse noise present in the image during transformation. The final denoised image is obtained.

Wavelets work for decomposing signals (such as images) into hierarchy of increasing resolutions. The advantage of wavelet denoising is possible to remove the noise with little loss of details. The wavelet mode denoises only the Gaussian type of noise. So when multiple noise present in the image it will remove only Gaussian the remaining noise are unremoved. So for removing the remaining noise and to preserve the fine details CWM filter is applied. The advantage of center weighted median filter can denoise the large window size. The proposed method consist of the following process

\section{EXPERIMENTAL RESULTS}

DICOM medical images are taken as test images for evaluating results. Here the average of ten images is taken for 
evaluation. The algorithm is tested in MATLAB (7.8 Version).The reconstruction of an image has the dimensions of 256 pixel intensity. The DICOM images contain a wide variety of subject matters and textures. Most of the images used are brain images with defect and without defect images. The PSNR and UQI value must be high for a medical image, MSE and ET must be less value for a better filtering algorithm.

\section{A. Peak Signal to Noise Ratio}

The PSNR is defined in logarithmic scale, in db (decibels). The table1 shows the parametric evaluation for mixed noise removal .The image metric PSNR is defined as:

$$
P S N R=20 . \log _{10}\left(\frac{M A X_{I}}{\sqrt{M S E}}\right)
$$

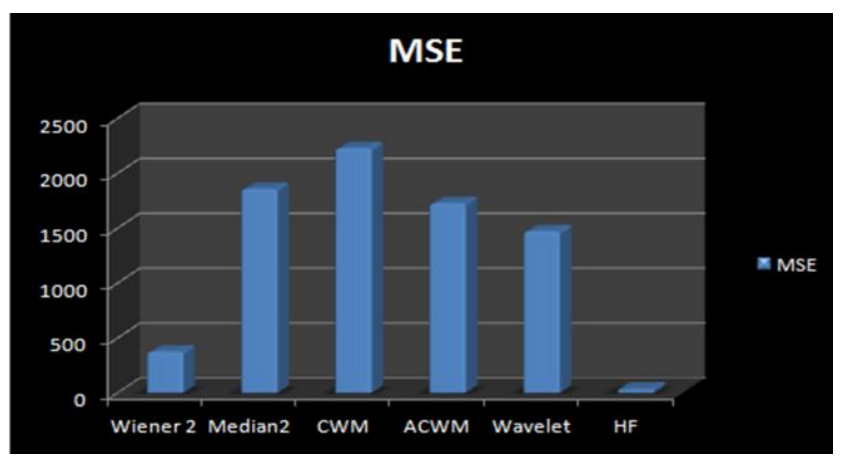

Figure 4. MSE value for Denoising Mixed noise

TABLE I. PARAMETRIC EVALUATION OF MIXED NOISE REMOVAL

\begin{tabular}{crrrr}
\hline Method & \multicolumn{1}{c}{ PSNR } & \multicolumn{1}{c}{ MSE } & \multicolumn{1}{c}{ UQI } & \multicolumn{1}{c}{ ET } \\
Wiener2 & 20.42063 & 375.333 & 0.7898 & 3.042129 \\
Median2 & 15.47557 & 1857.474 & 0.27858 & 2.929375 \\
CWM & 14.6842 & 2228.741 & 0.21503 & 3.62574 \\
ACWM & 15.79021 & 1727.661 & 0.35028 & 3.01658 \\
Wavelet & 71.2354 & 1472.231 & 0.231 & 3.2456 \\
HF & 21.49234 & 34.84196 & 0.98692 & 2.434407
\end{tabular}

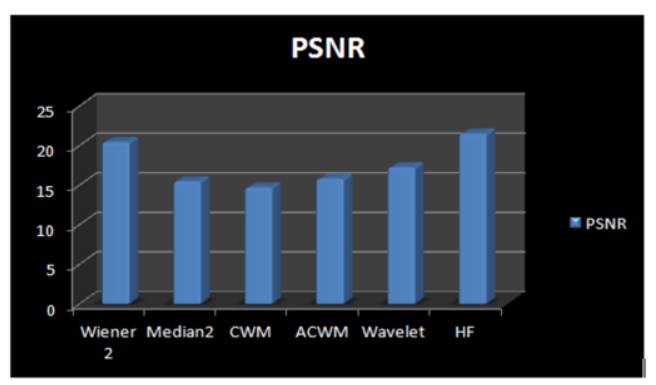

Figure 5. PSNR value for Denoising Mixed noise

The figure 4 shows the PSNR value for denosing mixed noise using didfferemt filtering technique. The PSNR value must be high for an image. The proposed method gives desire results when compare to other filtering technique.

\section{B. Mean Square Error (MSE):}

The metric MSE is defined as:

$M S E=\frac{1}{m n} \sum_{i=0}^{m-1} \sum_{j=0}^{n-1}\|I(i, j)-K(i, j)\|^{2}$

For two $m \times n$ monochrome images $I$ and $K$, one of the images is considered a noisy approximation of the other. The figure 5 shows the MSE value for denosing mixed noise using didfferemt filtering technique. The MSE value must be low for an better quality image. The propsed method gives suitable results when compare to other filtering technique.

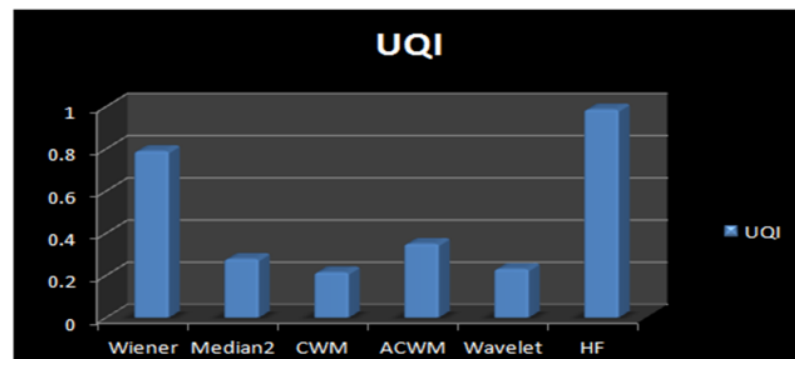

Figure 6. UQI value for Denoising Mixed noise

C. $U Q I$

UQI measures image similarity across distortion types. Distortions in UQI are measured as a combination of three factors; Loss of correlation, Luminance distortion and Contrast distortion. Let $\left\{\mathrm{x}_{\mathrm{i}}\right\}$ and $\left\{\mathrm{y}_{\mathrm{i}}\right\}=1,2, \ldots, \mathrm{N}$ be the original and the test image signals, respectively. The universal quality index is defined as

$$
U Q I=\frac{{ }^{4} \sigma_{x y} \overline{x y}}{\left[\sigma_{x}^{2}+\sigma_{x}{ }_{x}\right]\left[(\bar{x})^{2}+(\bar{y})^{2}\right]}
$$

The above figure 6 shows the UQI value for denosing mixed noise using didfferemt filtering technique

The UQI value must be high for an good image.

The propsed method gives most suitable results when compare to other filtering technique.The dynamic range of UQI is $[-1,1]$.

\section{Evaluation Time}

Evaluation Time (ET) of a filter is defined as the time taken by a digital computing platform to execute the filtering algorithms. The execution time taken by a filter should be low for online and real-time image processing applications.

Hence, a filter with lower ET is better than a filter having higher ET value when all other performance-measures are identical. 


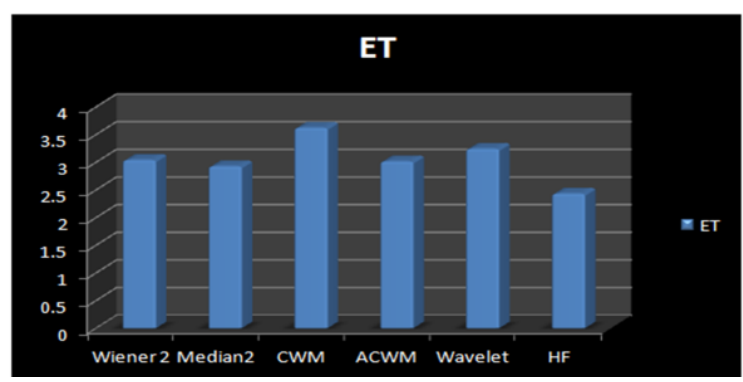

Figure 7. ET value for Denoising Mixed noise

The above figure 7 shows the ET value for denosing mixed noise using differemt filtering technique. The proposed method gives desirable results when compare to other filtering algorithm.

\section{CONCLUSION}

In this paper, an important research challenge is to improve the visual quality of $\mathrm{CT}$ brain images through image processing in order to detect abnormal brain at an early stage. This paper describes new methods for brain image preprocessing for noise suppression based on the wavelet transform. The image pre-processing was designed to suppress the noise present in local dense regions adaptively.

A new technique based on the hybridization of wavelet and center weighted median filters for denoising of variety of noisy images is presented in this paper. The model is experimented on standard Digital Imaging and Communications in Medicine (DICOM) images and the performances are evaluated in terms of peak signal to noise ratio (PSNR), Mean Absolute Error (MAE), Universal Image Quality Index (UQI) and Evaluation Time (ET). Results prove that utilization of center weighted median filters in combination with wavelet thresholding filters on DICOM images deteriorates the performance. The proposed filter gives suitable results on the basis of PSNR, MSE, UQI and ET. In addition, the proposed filter gives nearly uniform and consistent results on all the test images.

\section{REFERENCES}

[1] Prof. Syed Amjad Ali, Dr. Srinivasan Vathsal and Dr. K. Lal kishore,” CT Image Denoising Technique using GA aided Window-based Multiwavelet Transformation and Thresholding with the Incorporation of an Effective Quality Enhancement Method", International Journal of Digital Content Technology and its Applications, Vol. 4, N0. 4, P.75-87, 2010.

[2] Susmitha Vekkot, and Pancham Shukla, "A Novel Architecture for Wavelet based Image Fusion," World Academy of Science, Engineering and Technology, No. 57, P.372-377, 2009..

[3] Jenny Rajan, M.R Kaimal †Dept. Of Computer Science, University of

Kerala, Trivandrum, India "Image Denoising Using Wavelet Embedded anisotropic Diffusion", Appeared in the Proceedings of IEE International Conference on Visual Information Engineering, P.589 - 593, 2006

[4] Mukesh C. Motwani, Mukesh C. Gadiya, Rakhi C. Motwani "survey of image denoising techniques", Proceedings of GSPx 2004, Santa Clara Convention Center, Santa Clara, CA. P.27-30, 2004.

[5] Sudipta Roy, Nidul Sinha \& Asoke K. Sen," A New Hybrid image denoising method," International Journal of Information Technology and Knowledge Management, Vol. 2, No. 2, P. 491-497,2010.

[6] Mamta Juneja, Parvinder Singh Sandhu, "Design and Development of an Improved Adaptive Median Filtering Method for Impulse Noise Detection," International Journal of Computer and Electrical Engineering, Vol. 1, No. 5, P. 1793-8163,2009.

[7] S.Satheesh, Dr.KVSVR Prasad, "Medical Image Denoising using Adaptive Threshold based on Contourlet Transform," Advanced Computing: An International Journal ( ACIJ ), Vol.2, No.2,P.52-58, 2011.

[8] Geoffrine Judith.M.C1 and N.Kumarasabapathy, "Study and Analysis of Impulse Noise Reduction Filters," Signal \& Image Processing : An International Journal(SIPIJ) Vol.2, No.1,P. 82-92, 2011.

[9] Rastislav Lukac_, Bogdan Smołka, "Application of the Adaptive Center-Weighted Vector Median Framework for the Enhancement of CDNA Microarray Images,” Int. J. Appl. Math. Comput. Sci., Vol. 13, No. 3, 369-383, 2003.

[10] Amir Beck, Marc Teboulle, "A Fast Iterative Shrinkage-Thresholding Algorithm with Application to Wavelet-based Image Deblurring," IEEE conference, ICASSP 2009,P.693-696,2009.

[11] Naga Sravanthi Kota, G.Umamaheswara Reddy ," Fusion Based Gaussian noise Removal in the Images Using Curvelets and Wavelets With Gaussian Filter," International Journal of Image Processing (IJIP), Vol.5, No.4 ,P.456-468, 2011.

[12] Gnanambal Ilango and R. Marudhachalam, "New Hybrid Filtering Techniques for Removal of Gaussian Noise From Medical Images," ARPN Journal of Engineering and Applied Sciences, VOL. 6, NO. 2, P.8-12, 2011.

[13] Florian Luisier, Thierry Blu, Senior and Michael Unser, "Image Denoising in Mixed Poisson-Gaussian Noise," IEEE Transactions on Image Processing, Vol. 20, No. 3,P. 696-707, 2011.

[14] Pierre Gravel, Gilles Beaudoin, and Jacques A. De Guise, "A Method for Modeling Noise in Medical Images," IEEE Transactions on Medical Imaging, Vol. 23, No. 10,P.1221-1232, 2004.

\section{AUTHORS PROFILE}
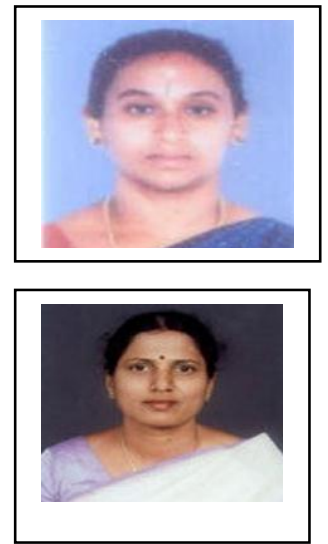

Ms. J.Umamaheswari Research Scholar in Computer Science, Dr. G.R.D college, Coimbatore. She has 5 years of teaching experience and two years in Research. Her areas of interest include Image Processing, Multimedia and communication. She has more than 3 publications at International level. She is a life member of professional organization IAENG.

Dr. G. Radhamani Professor \& Director in School of Computer Scienec, Dr.G.R..D College, Coimbatore. She was awarded "Bharat Joythi" in lieu of the yeomen services rendered with excellence in their respective fields. She has worked for the Malaysia Venture Capital Management Ph.d, and has been a reviewer for IEEE Wireless 